Nanopatterning of the (001) surface of crystalline Ge by ion irradiation at off-normal incidence : Experiment and simulation

\title{
Erb, Denise
}

$2020-10-26$

Erb , D , de Schultz , R , llinov , A , Nordlund , K, Bradley , R M \& Facsko , S 2020 , ' Nanopatterning of the (001) surface of crystalline Ge by ion irradiation at off-normal incidence : Experiment and simulation ' , Physical Review B , vol. 102 , no. 16 , 165422 . https://doi.org/10.1103/Phy

http://hdl.handle.net/10138/321565

https://doi.org/10.1103/PhysRevB.102.165422

unspecified

publishedVersion

Downloaded from Helda, University of Helsinki institutional repository.

This is an electronic reprint of the original article.

This reprint may differ from the original in pagination and typographic detail.

Please cite the original version. 


\title{
Nanopatterning of the (001) surface of crystalline Ge by ion irradiation at off-normal incidence: Experiment and simulation
}

\author{
Denise Erb $\odot,{ }^{1, *}$ Ricardo de Schultz $\odot,{ }^{1,2}$ Andrey Ilinov, ${ }^{3}$ Kai Nordlund ${ }^{3}$ R. Mark Bradley $\odot,{ }^{4}$ and Stefan Facsko $\oplus^{1, \dagger}$ \\ ${ }^{1}$ Ion Beam Center, Institute of Ion Beam Physics and Materials Research, \\ Helmholtz-Zentrum Dresden-Rossendorf, 01328 Dresden, Germany \\ ${ }^{2}$ Technische Universität Dresden, 01069 Dresden, Germany \\ ${ }^{3}$ Department of Physics, University of Helsinki, Helsinki 00014, Finland \\ ${ }^{4}$ Department of Physics, Colorado State University, Fort Collins, Colorado 80523, USA
}

(Received 15 May 2020; revised 29 September 2020; accepted 6 October 2020; published 26 October 2020)

\begin{abstract}
Intricate topographical patterns can form on the surface of crystalline $\mathrm{Ge}(001)$ subject to low-energy ion irradiation in the reverse epitaxy regime, i.e., at elevated temperatures which enable dynamic recrystallization. We compare such nanoscale patterns produced by irradiation from varied polar and azimuthal ion incidence angles with corresponding calculated surface topographies. To this end, we propose a continuum equation including both anisotropic erosive and anisotropic diffusive effects. Molecular dynamics simulations provide the coefficients of angle-dependent sputter erosion for the calculations. By merely changing these coefficients accordingly, the experimentally observed surface morphologies can be reproduced, except for extreme ion incidence angles. Angle-dependent sputter erosion is thereby identified as a dominant mechanism in ion-induced pattern formation on crystalline surfaces under irradiation from off-normal incidence angles.
\end{abstract}

DOI: 10.1103/PhysRevB.102.165422

\section{INTRODUCTION}

A solid surface subject to broad-beam low-energy ion irradiation is a complex nonequilibrium system, in which a number of different processes act simultaneously on the nanometer scale. These processes can be categorized as erosive, ballistic, or diffusive. While erosive processes remove material from the surface, ballistic and diffusive processes determine how atoms and vacancies are redistributed on the surface. An erosive, ballistic, or diffusive process can result in smoothing or in destabilization of the surface, depending on the given experimental conditions with respect to surface structure and temperature, ion mass and energy, or the incidence direction of the ion beam. Consider for instance these processes:

a. Erosive. The surface is eroded by sputtering, where shadowing effects and a locally varying incidence angle on nonplanar surfaces can cause heterogeneous erosion. On the one hand, for sufficient sputtering yield at low to intermediate ion incidence angles, the erosion rate depends on the local surface gradient such that tilted areas are eroded faster than planar areas-which results in overall smoothing of the surface (see, e.g., [1] and references therein). On the other hand, the erosion rate is dependent on the local surface curvature in such a way that concave areas are eroded faster than convex areas. Effectively, height variations are thereby amplified and the surface is destabilized [2].

b. Ballistic. The ion impacts relocate adatoms on the surface via momentum transfer. If the ion incidence direction is

\footnotetext{
*d.erb@hzdr.de

${ }^{\dagger}$ www.hzdr.de/ibc
}

close to the average surface normal, the momentum transfer from impinging ions will dominantly relocate surface atoms in a downhill direction - the surface will be smoothed. For oblique ion incidence at angles exceeding a critical value, however, surface atoms can be relocated in the uphill direction-height differences will be amplified [3].

c. Diffusive. Ion impacts result in the production of both adatoms and vacancies as mobile species, which then diffuse on the surface. The ion beam direction and the structural properties of the surface on the atomic scale can induce anisotropies in lateral mass transport. On an amorphous surface, thermal diffusion is isotropic and will tend to smooth the surface in order to reduce surface free energy [4]. This can be further enhanced by an increased areal density of mobile surface vacancies and adatoms produced by ion irradiation. In contrast, on a crystalline surface, the diffusing surface vacancies and adatoms can encounter the Ehrlich-Schwoebel barrier [5] when trying to cross step edges, which results in an effective uphill mass current and thus in destabilization of the surface.

The simultaneous presence of counteracting smoothing and destabilizing mechanisms can result in the formation of periodic nanoscale patterns on the surface [2,6-9]. For ion energies in the range of $10^{2}$ to $10^{3} \mathrm{eV}$, this type of spontaneous surface nanopatterning has been observed on a wide variety of elemental and compound materials, including metals, semiconductors, and insulators. The pattern morphology depends on which surface processes are dominant under the given experimental conditions; various surface morphologies including parallel ripples with sinusoidal or sawtooth profile [10-14]; nanocone [15-17] or nanohole [18-20] patterns; and pyramidal structures with three-, four-, or sixfold symmetry $[14,21,22]$ have been reported. 


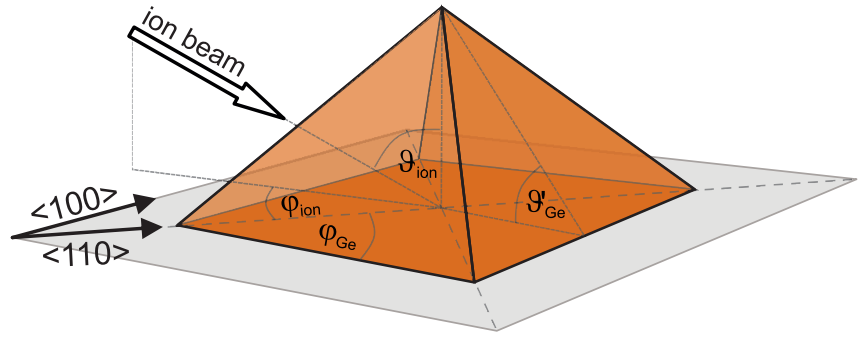

FIG. 1. Schematic drawing of a single pyramidal mound structure, showing the polar facet angle $\vartheta_{\mathrm{Ge}}^{\prime}$ and the azimuthal facet angle $\varphi_{\mathrm{Ge}}$. A direction of ion incidence is indicated by the broad white arrow, with the polar angle of incidence, $\vartheta_{\text {ion }}$, and the azimuthal angle of incidence, $\varphi_{\text {ion }}$.

With possible applications of this self-assembled surface pattern formation in bottom-up nanofabrication already being explored [23-26], there are still fundamental aspects to be clarified: For instance, the influence of the polar and azimuthal ion beam direction on the patterning morphology has been widely studied for amorphous or amorphized surfaces with isotropic diffusion, but a corresponding investigation is still lacking for crystalline surfaces with anisotropic diffusion in the reverse epitaxy regime [21] — and so is a theoretical description. In a continuation of other recent work [27], in this paper we present results on the dependence of the ion-induced nanoscale patterns on $\mathrm{Ge}(001)$ on both the polar and azimuthal angles of ion incidence. We compare atomic force microscopy (AFM) data of $\mathrm{Ge}(001)$ surfaces after ion irradiation with simulated surface topographies. The latter were obtained using a continuum equation approach with coefficients obtained from molecular dynamics (MD) simulations. By investigating the patterning resulting from irradiation from two specific azimuthal ion incidence angles, we are able to disentangle the components of anisotropic lateral diffusion in the $\langle 100\rangle$ and $\langle 110\rangle$ directions on the crystalline surface.

\section{EXPERIMENTAL PROCEDURES AND RESULTS}

Epi-ready Ge(001) surfaces were cleaned with ethanol and irradiated with $1 \mathrm{keV} \mathrm{Ar}^{+}$ions from a Kaufman-type ion source. The high-vacuum setup employed for irradiation had a base pressure of $10^{-7}$ mbar and a working gas pressure of $2.5 \times 10^{-4}$ mbar. During irradiation, the samples were held at a temperature of $380{ }^{\circ} \mathrm{C}$. All samples were irradiated with an effective ion flux of $1 \times 10^{15} \mathrm{~cm}^{-2} \mathrm{~s}^{-1}$ (with the ion current adapted to the polar incidence angle) and a fluence of $1 \times 10^{18} \mathrm{~cm}^{-2}$. After irradiation the samples were quenched to room temperature by immediately removing them from the heating stage. They were then transferred through air to a separate ultrahigh-vacuum setup with a base pressure better than $1 \times 10^{-9}$ mbar. There, the surface topographies were imaged via AFM performed with an Omicron VT SPM Lab instrument in contact mode, using Nanosensors PointProbe Plus cantilevers with a radius of $7 \mathrm{~nm}$. Each AFM topography micrograph had a scan range of $3 \mu \mathrm{m} \times 3 \mu \mathrm{m}$ and $375 \times$ 375 data points. The data were processed by means of the GWYDDION software package [28].

The angles are defined as follows (see also Fig. 1): $\vartheta_{\mathrm{Ge}}$ and $\varphi_{\mathrm{Ge}}$ denote the polar and azimuthal facet angles, respectively. $\vartheta_{\mathrm{Ge}}=0^{\circ}$ corresponds to the orientation of the macroscopic surface normal. The out-of-plane facet inclination is thus defined as $\vartheta_{\mathrm{Ge}}^{\prime}:=90^{\circ}-\vartheta_{\mathrm{Ge}} \cdot \vartheta_{\text {ion }}$ and $\varphi_{\text {ion }}$ denote the polar and azimuthal ion incidence angles, respectively. $\vartheta_{\text {ion }}=0^{\circ}$ corresponds to the orientation of the macroscopic surface normal. $\varphi_{\text {ion }}=0^{\circ}$ corresponds to the in-plane component of the incident ion beam direction being in the $\langle 110\rangle$ direction and $315^{\circ}$ corresponds to it being in the $\langle 100\rangle$ direction.

Keeping the samples at elevated temperature during ion irradiation enables dynamic recrystallization; the sample surfaces thereby remain crystalline throughout the irradiation procedure. The resulting morphology is expected to exhibit the pattern of alternating pyramid-shaped pits and mounds which is characteristic for $\mathrm{Ge}(001)$ in the reverse epitaxy regime of ion-induced pattern formation [21]. Irradiations
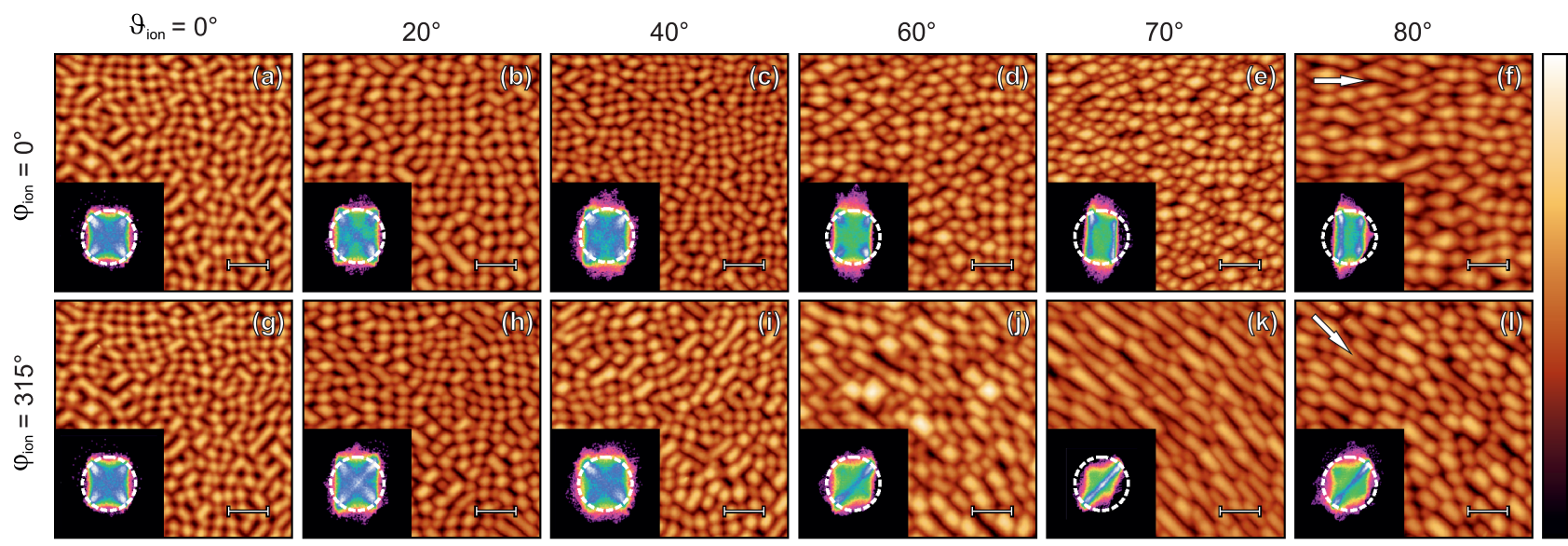

FIG. 2. AFM topography images of $\mathrm{Ge}(001)$ surfaces after ion irradiation from an azimuthal direction parallel to $\langle 110\rangle$ (top) and $\langle 100\rangle$ (bottom), respectively, as indicated by the arrows in (f) and (l). Samples were irradiated with $1 \times 10^{18} \mathrm{~cm}^{-2}$ of $1 \mathrm{keV} \mathrm{Ar}^{+}$ions. From left to right, the polar ion incidence angle increases from $\vartheta_{\text {ion }}=0^{\circ}$ to $80^{\circ}$ as labeled. The sample temperature was $380^{\circ} \mathrm{C}$ for all samples. All lateral scale bars are $500 \mathrm{~nm}$ long and the height scale range is (a), (g), (k) $25 \mathrm{~nm}$, (b), (c), (h-j) $30 \mathrm{~nm}$, and (d-f), (l) $35 \mathrm{~nm}$. The insets show corresponding distributions of the out-of-plane inclination and in-plane angular orientation of the surfaces, with the white ring indicating an out-of-plane inclination of $\vartheta_{\mathrm{Ge}}^{\prime}=10^{\circ}$. 

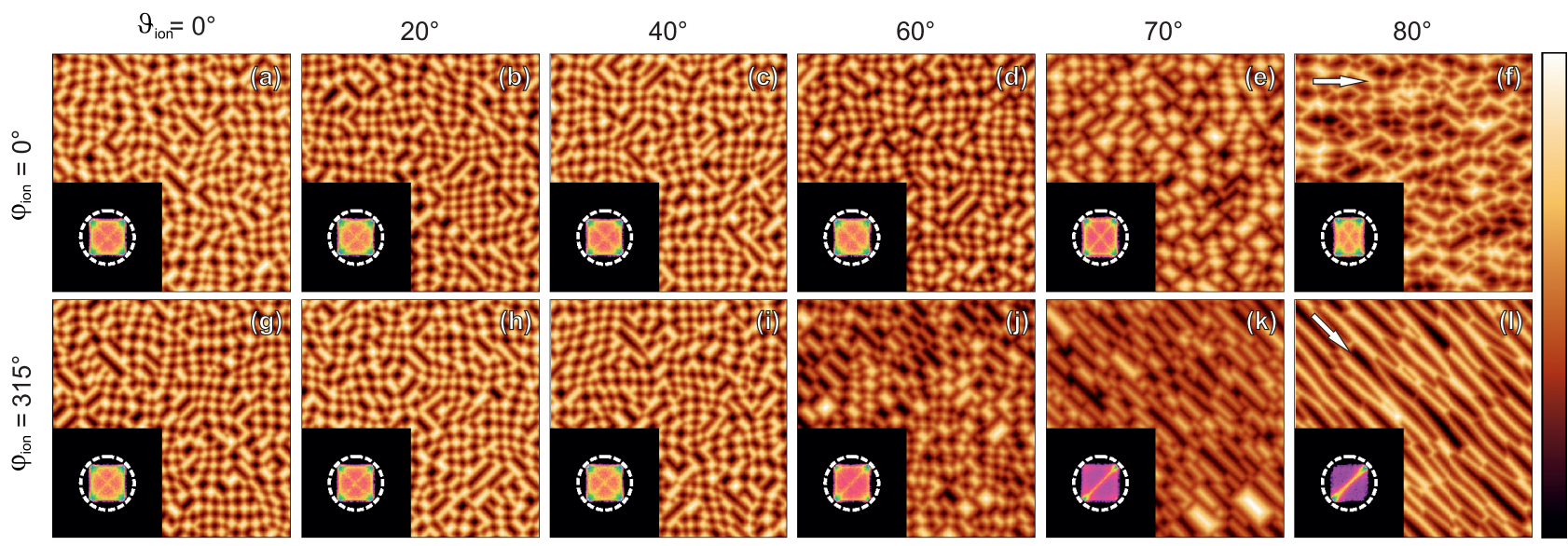

FIG. 3. Results of numerical integration of Eq. (2) with parameters as listed in Table I: simulated surface topographies for Ge(001) after ion irradiation from an azimuthal direction parallel to $\langle 110\rangle$ (top) and $\langle 100\rangle$ (bottom), respectively, at polar ion incidence angles $\vartheta_{\text {ion }}$ as indicated. The height scale range in arbitrary units is $(\mathrm{a}-\mathrm{c}),(\mathrm{g}-\mathrm{j}) 2$, (d), (f), (k), (l) 3, and (e) 4. The insets show corresponding distributions of the out-of-plane inclination and in-plane angular orientation of the surfaces, with the white ring indicating an out-of-plane inclination of $\vartheta_{\mathrm{Ge}}^{\prime}=10^{\circ}$.

were performed at normal ion incidence and at polar incidence angles of $\vartheta_{\text {ion }}=20^{\circ}, 40^{\circ}, 60^{\circ}, 70^{\circ}$, and $80^{\circ}$ for azimuthal ion incidence along the $\langle 100\rangle$ and $\langle 110\rangle$ directions. The resulting surface topographies were imaged by AFM and are depicted in Fig. 2. Figure 3 shows corresponding simulated surface topographies and is discussed in detail in Sec. III. The insets show the corresponding two-dimensional angular histograms.

For normal incidence irradiation, the Ge(001) surface exhibits a dense checkerboard pattern of pyramidal pits and mounds and contains no horizontal surface areas. These faceted structures have rectangular bases with edges in the in-plane $\langle 100\rangle$ and $\langle 010\rangle$ orientations and tilted sidewalls with an inclination of approximately $\vartheta_{\mathrm{Ge}}^{\prime}=8^{\circ}$ with respect to the macroscopic horizontal surface plane [14]. The length ratio of the edges is close to 1 for most structures, while fewer structures are elongated in $\langle 100\rangle$ or $\langle 010\rangle$ directions. For both azimuthal ion beam directions, we observe a progressively larger elongation of the structures along the directions of the ion beam with increasing polar ion incidence angle. To quantify how the surface morphology depends on azimuthal and polar irradiation directions, we extract histograms of the azimuthal facet angles $\varphi_{\mathrm{Ge}}$ (i.e., the in-plane facet orientation) from the AFM data. These are plotted in Figs. 4(a) and 4(e). Figure 5 shows the corresponding analysis results for the simulation data for comparison and is discussed in Sec. III.

For irradiation along $\langle 100\rangle$, i.e., $\varphi_{\text {ion }}=315^{\circ}$, the relative peak height, which is indicative of the relative surface area of facets with the given orientations, is strongly dependent on the ion incidence angle [Figs. 4(a) and 4(b)]: At normal ion incidence, the peaks corresponding to the four facets of the pyramidal structures are of the same relative height. With increasing polar ion incidence angle $\vartheta_{\text {ion }}$, the relative peak height increases approximately from 0.25 to 0.5 for facets 1 and 3, which at $\varphi_{\mathrm{Ge}}=45^{\circ}$ and $\varphi_{\mathrm{Ge}}=225^{\circ}$ are oriented parallel to the incident ion beam, while it decreases almost to zero for facets 2 and 4 , which at $\varphi_{\mathrm{Ge}}=135^{\circ}$ and $\varphi_{\mathrm{Ge}}=$ $315^{\circ}$ are oriented perpendicular to the incident ion beam. The peak positions, however, do not change significantly, indi- cating that the in-plane facet orientations $\varphi_{\mathrm{Ge}}$ and thereby the rectangular base shape of the pyramidal structures are preserved for all polar ion incidence angles $\vartheta_{\text {ion }}$ [Figs. 4(a) and 4(c)]. Thus, for off-normal ion incidence angles $\vartheta_{\text {ion }}$ the pyramidal surface structures elongate along the azimuthal ion incidence direction of $\langle 100\rangle$. An interesting exception from this general behavior is observed for $\vartheta_{\text {ion }}=40^{\circ}$, where the pyramidal structures are slightly elongated perpendicular to the azimuthal ion incidence orientation, resulting in the slight inversion of the trend in the dependence of the relative peak height on $\vartheta_{\text {ion }}$ seen in Fig. 4(b). The facet inclination [Fig. 4(d)] tends to remain constant for facets 2 and 4 (oriented perpendicular to the ion beam direction), and to decrease for facets 1 and 3 (oriented parallel to the ion beam direction).

For irradiation along $\langle 110\rangle$, i.e., $\varphi_{\text {ion }}=0^{\circ}$, both positions and heights of the histogram peaks depend on the polar ion incidence angle $\vartheta_{\text {ion }}$ [Fig. 4(e)]. The facet areas change as indicated by the relative peak heights [Fig. 4(f)]: At $\vartheta_{\text {ion }}=80^{\circ}$, the relative peak height is larger for facets 1 and 4 , which at $\varphi_{\mathrm{Ge}}=45^{\circ}$ and $\varphi_{\mathrm{Ge}}=315^{\circ}$ are facing the incident ion beam, while it is smaller for facets 2 and 3 , which at $\varphi_{\mathrm{Ge}}=135^{\circ}$ and $\varphi_{\mathrm{Ge}}=225^{\circ}$ are averted from the ion beam in the azimuthal direction. Simultaneously, the peak positions, indicating the facet orientations $\varphi_{\mathrm{Ge}}$, are changing notably by about $+20^{\circ}$ for facets 1 and 3 and by about $-20^{\circ}$ for facets 2 and 4 [Fig. 4(g)]. Thus, for irradiation along $\langle 110\rangle$ at large polar ion incidence angles $\vartheta_{\text {ion }}$ the pyramidal structures assume a distinctly rhomboidal base shape for $\vartheta_{\text {ion }} \geqslant 60^{\circ}$ and the facets facing the ion beam are of larger relative area than those on the opposite side. The out-of-plane inclination [Fig. 4(h)] tends to decrease for all facets.

\section{THEORETICAL DESCRIPTION AND SIMULATION RESULTS}

The development of local surface height $h(x, y, t)$ with time can be described by a deterministic continuum equation taking into account ballistic and diffusive mass currents as well as 

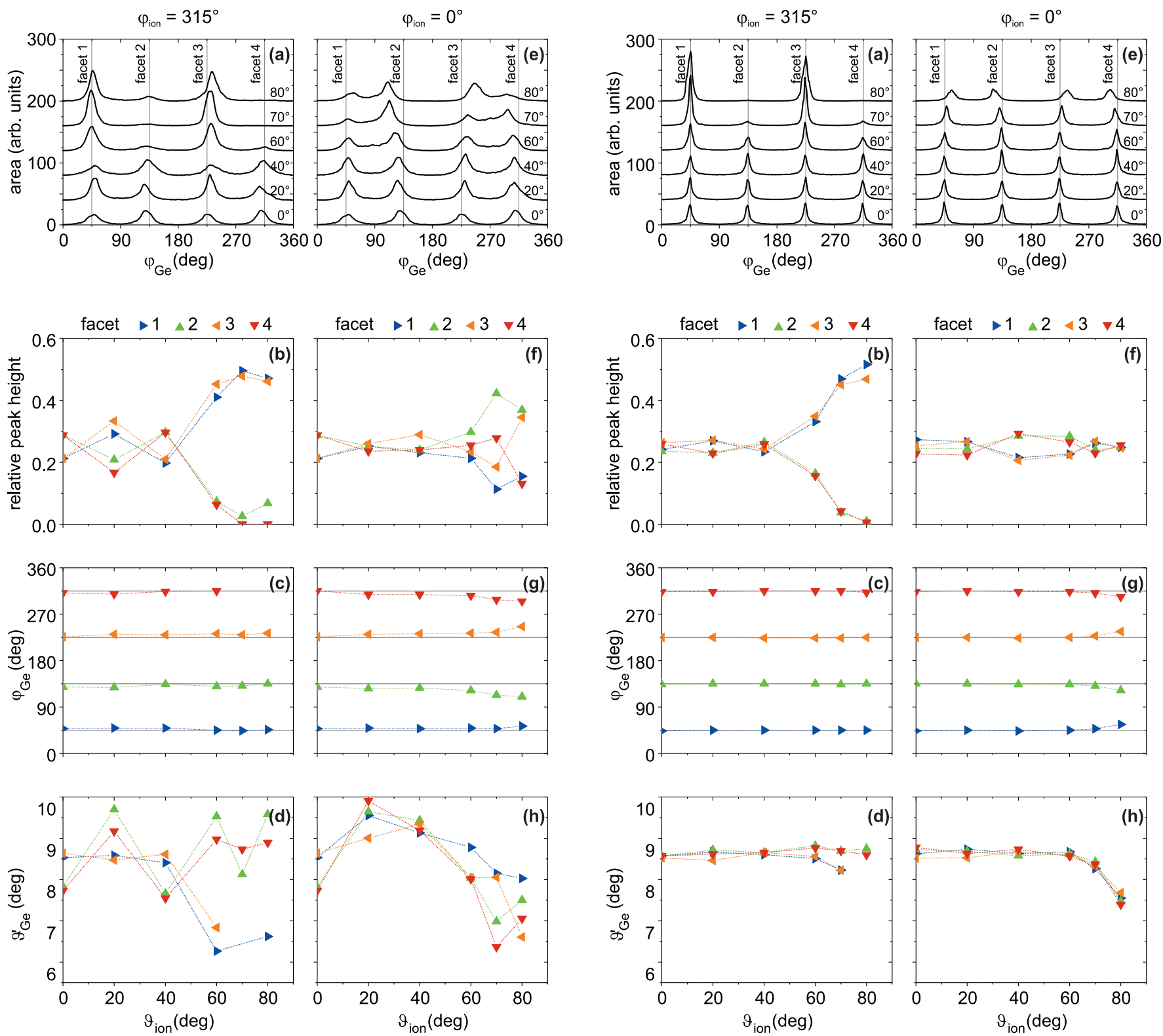

FIG. 4. Data extracted from the AFM measurements on the azimuthal and polar facet angles of the pyramidal structures for irradiations along (a)-(d) the $\langle 100\rangle$ direction, i.e., $\varphi_{\text {ion }}=315^{\circ}$, and (e)-(h) the $\langle 110\rangle$ direction, i.e., $\varphi_{\text {ion }}=0^{\circ}$. (a), (e) Histograms of the in-plane facet orientation $\varphi_{\mathrm{Ge}}$ for increasing polar ion incidence angle $\vartheta_{\text {ion. }}$. The curves are offset vertically for clarity. (b), (f) Relative heights of the peaks in (a) and (e), respectively, as functions of $\vartheta_{\text {ion }}$. (c), (g) In-plane orientations $\varphi_{\mathrm{Ge}}$ of the four facets of the pyramidal structures as functions of $\vartheta_{\text {ion }}$ (d), (h) Out-of-plane inclinations $\vartheta_{\mathrm{Ge}}^{\prime}$ of the four facets of the pyramidal structures as functions of $\vartheta_{\text {ion }}$.

erosive effects [29]. The isotropic equation suitable for normal ion incidence reads

$$
\partial_{t} h=-\mathrm{v}_{0}+v \nabla^{2} h+\lambda(\nabla h)^{2}-\nabla \cdot \vec{j}_{\text {diff }} .
$$

Here, $v_{0}$ is the constant erosion rate of a planar surface. $v \nabla^{2} h$ denotes the curvature-dependent sputter rate and ballistic mass redistribution according to the Bradley-Harper and Carter-Vishnyakov models [2,3]. The term $\lambda(\nabla h)^{2}$ accounts
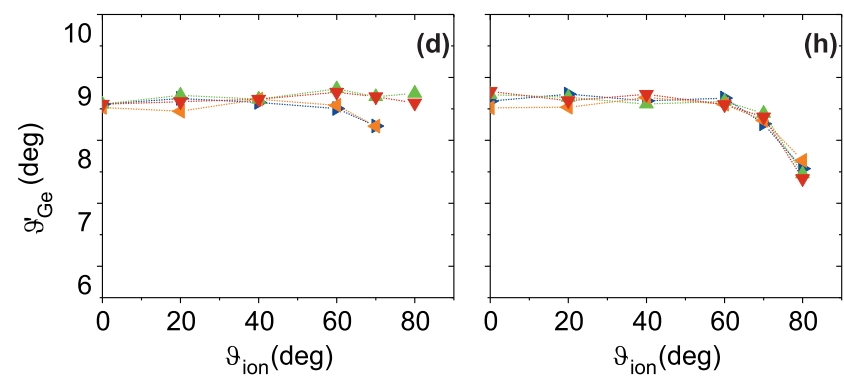

FIG. 5. Data extracted from the simulated surface topographies on the azimuthal and polar facet angles of the pyramidal structures for irradiations along (a)-(d) the $\langle 100\rangle$ direction, i.e., $\varphi_{\text {ion }}=315^{\circ}$, and (e)-(h) the $\langle 110\rangle$ direction, i.e., $\varphi_{\text {ion }}=0^{\circ}$. (a), (e) Histograms of the in-plane facet orientation $\varphi_{\mathrm{Ge}}$ for increasing polar ion incidence angle $\vartheta_{\text {ion }}$. The curves are offset vertically for clarity. (b), (f) Relative heights of the peaks in (a) and (e), respectively, as functions of $\vartheta_{\text {ion }}$. (c), (g) In-plane orientations $\varphi_{\mathrm{Ge}}$ of the four facets of the pyramidal structures as functions of $\vartheta_{\text {ion }}$. (d), (h) Out-of-plane inclinations $\vartheta_{\mathrm{Ge}}^{\prime}$ of the four facets of the pyramidal structures as functions of $\vartheta_{\text {ion }}$.

for the tilt-dependent sputter rate [30,31] and the mass current $\vec{j}_{\text {diff }}$ describes diffusive mass transport. In order to take the varied polar and azimuthal ion beam directions into account, we employ the anisotropic continuum equation [32]

$$
\begin{aligned}
\partial_{t} h= & -\mathrm{v}_{0}+v_{x}^{\prime} \partial_{x x} h+v_{y}^{\prime} \partial_{y y} h+v_{x y}^{\prime} \partial_{x y} h \\
& +\lambda_{x}^{\prime}\left(\partial_{x} h\right)^{2}+\lambda_{y}^{\prime}\left(\partial_{y} h\right)^{2}+\lambda_{x y}^{\prime}\left(\partial_{x} h \cdot \partial_{y} h\right) \\
& -\nabla \cdot \vec{j}_{\text {diff }}
\end{aligned}
$$


with the following coefficients [33]:

$$
\begin{aligned}
v_{x}^{\prime} & =\frac{v_{x}+v_{y}}{2}+\frac{v_{x}-v_{y}}{2} \cos \left(2 \varphi_{\text {ion }}\right), \\
v_{y}^{\prime} & =\frac{v_{x}+v_{y}}{2}-\frac{v_{x}-v_{y}}{2} \cos \left(2 \varphi_{\text {ion }}\right), \\
v_{x y}^{\prime} & =\frac{v_{x}-v_{y}}{2} \sin \left(2 \varphi_{\text {ion }}\right), \\
\lambda_{x}^{\prime} & =\frac{\lambda_{x}+\lambda_{y}}{2}+\frac{\lambda_{x}-\lambda_{y}}{2} \cos \left(2 \varphi_{\text {ion }}\right), \\
\lambda_{y}^{\prime} & =\frac{\lambda_{x}+\lambda_{y}}{2}-\frac{\lambda_{x}-\lambda_{y}}{2} \cos \left(2 \varphi_{\text {ion }}\right), \\
\lambda_{x y}^{\prime} & =\frac{\lambda_{x}-\lambda_{y}}{2} \sin \left(2 \varphi_{\text {ion }}\right),
\end{aligned}
$$

where $v_{x, y}$ and $\lambda_{x, y}$ are the coefficients for the case $\varphi_{\text {ion }}=0$, and $\langle 110\rangle$ is defined as the $x$ direction. The mass current $\vec{j}_{\text {diff }}$ is comprised of three terms describing thermally driven diffusion [21]:

$$
\begin{aligned}
\vec{j}_{\text {diff }} & =\vec{j}_{\mathrm{cKPZ}}+\vec{j}_{\text {iso }}+\vec{j}_{\mathrm{ES}} \\
& =\sigma \nabla(\nabla h)^{2}+\kappa \nabla\left(\nabla^{2} h\right)+\left[\begin{array}{l}
\Omega_{1} \partial_{x} h-\Omega_{3}\left(\partial_{x} h\right)^{3} \\
\Omega_{2} \partial_{y} h-\Omega_{4}\left(\partial_{y} h\right)^{3}
\end{array}\right] .
\end{aligned}
$$

The mass-conserving Kardar-Parisi-Zhang term $\vec{j}_{\text {cKPZ }}$ describes a nonlinear isotropic mass current which can break the up-down symmetry of the surface pattern [34]. $\vec{j}_{\text {iso }}$ is taken to be an isotropic, thermally activated diffusion current, which leads to smoothing of the surface. $\vec{j}_{\mathrm{ES}}$ accounts for anisotropic diffusion due to the Ehrlich-Schwoebel (ES) and kink barriers, i.e., biased diffusion across terrace steps and around kinks on a crystalline surface [5,35-37]. This term results in net uphill mass currents and the formation of surface facets with angular orientations for which this mass current becomes zero $[21,38]$.

The coefficients $\lambda_{x}$ and $\lambda_{y}$ for angle-dependent sputter erosion can be expressed analytically [39] as

$$
\begin{aligned}
& \lambda_{x} \propto-F^{\prime \prime}\left(\vartheta_{\text {ion }}\right)-F\left(\vartheta_{\text {ion }}\right), \\
& \lambda_{y} \propto-\frac{F^{\prime}\left(\vartheta_{\text {ion }}\right)}{\tan \left(\vartheta_{\text {ion }}\right)}-F\left(\vartheta_{\text {ion }}\right),
\end{aligned}
$$

with

$$
F\left(\vartheta_{\text {ion }}\right)=\cos \left(\vartheta_{\text {ion }}\right) Y\left(\vartheta_{\text {ion }}\right),
$$

where $Y\left(\vartheta_{\text {ion }}\right)$ is the angle-dependent sputtering yield. $Y\left(\vartheta_{\text {ion }}\right)$ was obtained following the general principles outlined in [40] and using the open-source PARCAS MD code [41,42]. For this study, the $1 \mathrm{keV} \mathrm{Ar}$ atoms were shot at $\mathrm{Ge}(001)$ surfaces on random positions near the center, at various polar incidence angles and with azimuthal directions according to the experimental ones. The Ge surface was modeled with the modified Stillinger-Weber-like potential for Ge introduced in [41] in simulation cells that contained $20 \times 20 \times 12$ unit cells of atoms. As usual in collision cascade calculations, an adaptive time step was used [43] and temperature was controlled only at the borders of the simulation cell. Electronic stopping was applied on the ion and atoms inside the simulation cell with kinetic energy exceeding $5 \mathrm{eV}$. For each incoming angle, 300 ions were simulated to obtain a sputtering yield with reasonably

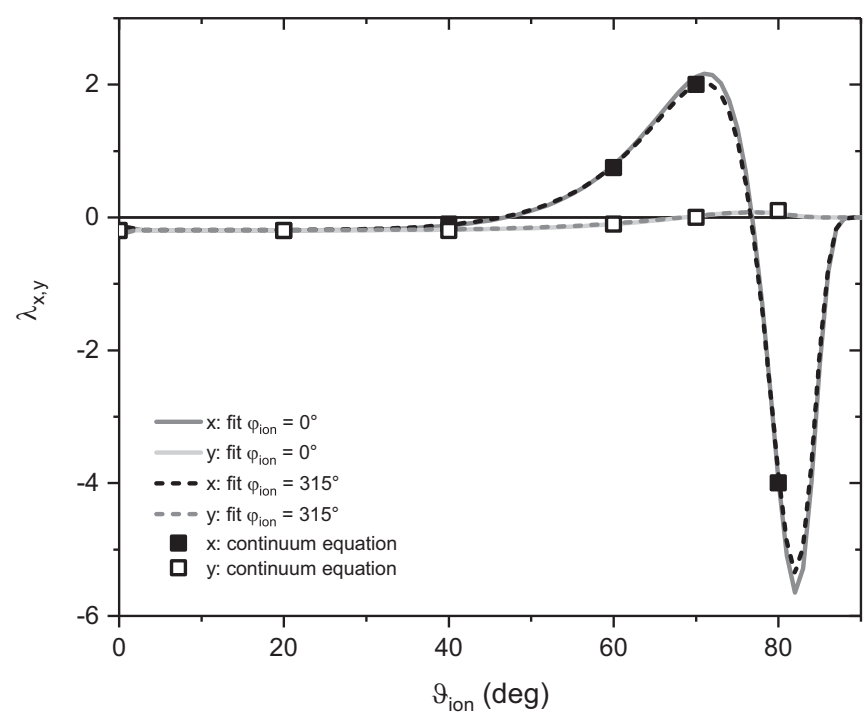

FIG. 6. Dependence of $\lambda_{x, y}$ on the ion incidence angle $\vartheta_{\text {ion }}$ as derived from fitting the angle-dependent sputter yield by Eq. (7) (lines) and values of $\lambda_{x, y}$ used for simulating the surface topographies shown in Fig. 3 (symbols).

small statistical uncertainty. $Y\left(\vartheta_{\text {ion }}\right)$ was fitted by the empirical Yamamura sputter yield function [44]

$$
Y\left(\vartheta_{\text {ion }}\right)=\frac{\exp \left(f_{1}-\frac{f_{1}}{\cos \left(\vartheta_{\text {ion }}\right)}\right)}{\cos ^{f_{2}}\left(\vartheta_{\text {ion }}\right)}
$$

with $f_{1}=1.0019$ and $f_{2}=2.741$ for $\varphi_{\text {ion }}=0^{\circ}, f_{1}=1.0047$ and $f_{2}=2.708$ for $\varphi_{\text {ion }}=315^{\circ}$. The coefficients $\lambda_{x}$ and $\lambda_{y}$ are thus almost the same for the two different azimuthal beam angles. Figure 6 shows these coefficients as functions of the polar ion incidence angle $\vartheta_{\text {ion }}$, obtained from the fit to the MD simulations via Eq. (5). The values of $\lambda_{x}$ and $\lambda_{y}$ do not deviate much from zero and $\lambda_{x} \approx \lambda_{y}$ for smaller angles. Consequently, for these angles also the pattern morphology does not deviate much from the morphology obtained at normal incidence. However, for higher angles, $\lambda_{x}$ is large enough in comparison to $\lambda_{y}$ to cause a substantial change of the surface morphology (see Fig. 3).

Equation (1) is a variant of the continuum equation first proposed by Cuerno and Barabási [29]. Being valid for oblique incidence, the equation by Cuerno and Barabási featured anisotropic terms for angle-dependent sputter erosion as well as curvature-dependent sputtering and ballistic mass redistribution. However, it did not account for the anisotropic diffusion induced by a crystalline surface. Ou et al. [21] then employed a variant of this equation limited to the case of normal ion incidence, where they introduced the anisotropic diffusive term accounting for the effect of the ES barrier to first describe patterning of $\mathrm{Ge}(001)$ in the reverse epitaxy regime. In order to include the additional effects of nonnormal ion incidence, we use the anisotropic form of this equation as given in Eq. (2), where the coefficients $\lambda_{x}\left(\vartheta_{\text {ion }}\right)$ and $\lambda_{y}\left(\vartheta_{\text {ion }}\right)$ describe angle-dependent anisotropic erosion. By means of numerical integration of Eq. (2) $[9,32,45]$, we simulated the surface topographies of crystalline $\mathrm{Ge}(001)$ resulting from ion irradiation at two azimuthal directions and 
TABLE I. Parameters for simulating the surface patterns in Fig. 3 via numerical integration of Eq. (2). In all simulations $v_{x, y}=0, \sigma=0$, $\kappa=1, \Omega_{1,2}=1$, and $\Omega_{3,4}=80$.

\begin{tabular}{lcc}
\hline \hline$\vartheta_{\text {ion }}(\mathrm{deg})$ & $\lambda_{x}$ & $\lambda_{y}$ \\
\hline 0 & -0.2 & -0.2 \\
20 & -0.2 & -0.2 \\
40 & -0.1 & -0.2 \\
60 & 0.75 & -0.1 \\
70 & 2.0 & 0.0 \\
80 & -4.0 & 0.1 \\
\hline
\end{tabular}

different polar incidence angles. The simulation started from a surface with uncorrelated low-amplitude roughness and lasted for 25000 time units. We chose $v_{x, y}=0, \sigma=0, \kappa=1, \Omega_{1}=$ $\Omega_{2}=1, \Omega_{3}=\Omega_{4}=80$, and $\lambda_{x}\left(\vartheta_{\text {ion }}\right), \lambda_{y}\left(\vartheta_{\text {ion }}\right)$ as listed in Table I, according to the dependency shown in Fig. 6. Transformation to a moving reference frame allows us to eliminate the term $v_{0}$ describing a homogeneous erosion of the entire surface at constant rate; it is therefore not considered further. Setting $v_{x, y}=0$ neglects any curvature-dependent sputtering and ballistic mass redistribution. We consider this justified for the case of $\mathrm{Ge}$ irradiated with $1 \mathrm{keV} \mathrm{Ar}^{+}$ions, because the characteristic ripple patterns resulting from curvaturedependent sputtering and ballistic mass redistribution are not observed: in this system, instead the surface remains smooth when irradiated at room temperature (see, e.g., [46] as well as [32]). Choosing $\sigma=0$ in the cKPZ term corresponds to disregarding diffusive effects as a source for possible up-down asymmetry. A previous publication [21] included this term in the continuum equation and showed how diffusion leads to up-down symmetry breaking when increasing the surface temperature, while erosive effects were of lesser interest then. Here, we focus on erosive effects for angle-dependent erosion. The polar facet angle observed experimentally is reproduced by setting $\Omega_{1}=\Omega_{2}=1$ and $\Omega_{3}=\Omega_{4}=80$.

The simulated topographies for a series of polar ion incidence angles are shown in Fig. 3, where the insets display the corresponding two-dimensional angular histograms of the surface height. We find good qualitative agreement with the experimental results shown in Fig. 2 for the range of polar incidence angles $\vartheta_{\text {ion }}=0^{\circ}-70^{\circ}$. For $\vartheta_{\text {ion }}=80^{\circ}$, however, the topography appears inverted, and especially for $\varphi_{\text {ion }}=315^{\circ}$ the simulated structures are much more elongated than the experimentally observed ones. Results from experiment and simulation also agree well quantitatively: Data extracted from the simulations are plotted in Fig. 5. The series of histograms shows the same trend of changing relative peak height for irradiation with $\varphi_{\text {ion }}=315^{\circ}$ and changing peak position for irradiation with $\varphi_{\text {ion }}=0^{\circ}$. The change in relative peak height is even quantitatively very well reproduced for $\varphi_{\text {ion }}=315^{\circ}$. For $\varphi_{\text {ion }}=0^{\circ}$, however, the relative peak heights obtained from the simulation remain constant, while they are dependent on $\vartheta_{\text {ion }}$ in experiment. Also the out-of-plane facet inclinations of the simulated surfaces follow the same general trend as seen in the experimental data for both irradiation directions in Figs. 4(d) and 4(h). Figure 7 shows a comparison of the characteristic length (defined as the position of the first maximum
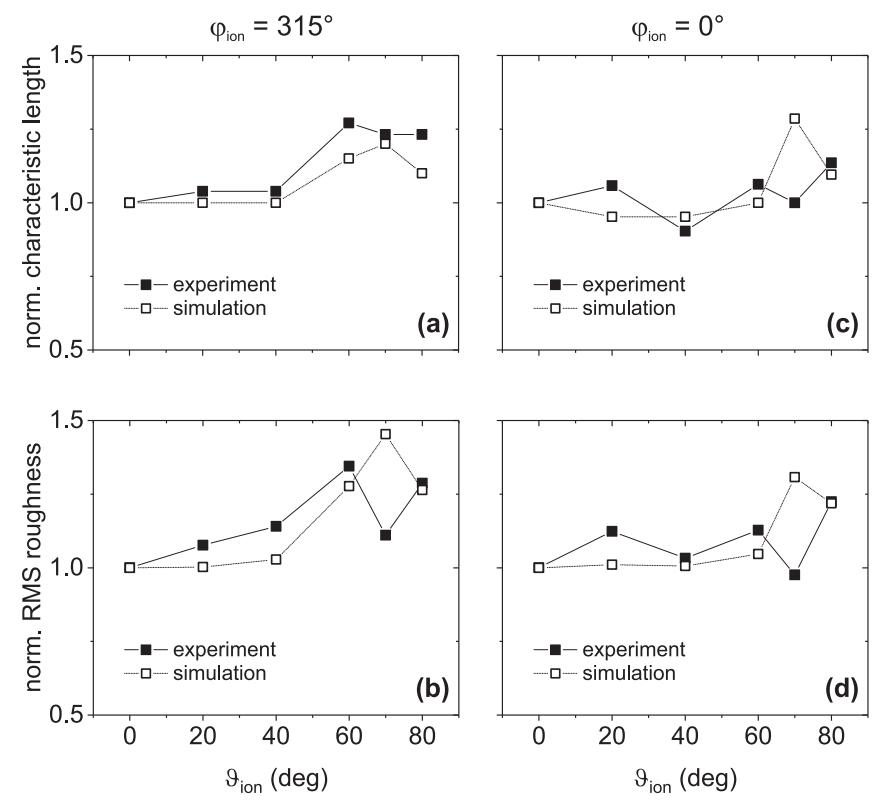

FIG. 7. Dependence of the normalized characteristic length and the root-mean-square roughness of the patterned surfaces on the polar ion incidence angle $\vartheta_{\text {ion }}$. (a), (b) Irradiation along $\langle 100\rangle$, i.e., $\varphi_{\text {ion }}=315^{\circ}$; (c), (d) irradiation along $\langle 110\rangle$, i.e., $\varphi_{\text {ion }}=0^{\circ}$. Solid (open) symbols show data obtained from experiments (simulations). Lines are guides to the eye.

in the radial autocorrelation function) and the root-meansquare roughness, both normalized to the respective values at normal ion incidence, for the experimentally and numerically obtained surfaces. The simulations generally reproduce the increase of both quantities with the polar angle of ion incidence with good agreement. However, for $\vartheta_{\text {ion }}=70^{\circ}$ the simulated values are significantly higher than the experimental ones, except for the normalized characteristic length for $\varphi_{\text {ion }}=315^{\circ}$. The agreement for $\vartheta_{\text {ion }}=80^{\circ}$ then appears better; it should be noted though that the surfaces shown in Figs. 2 and 3 display pronounced morphological differences for this polar incidence angle.

\section{DISCUSSION}

We find that the anisotropic continuum equation

$$
\begin{aligned}
\partial_{t} h= & \lambda_{x}^{\prime}\left(\partial_{x} h\right)^{2}+\lambda_{y}^{\prime}\left(\partial_{y} h\right)^{2}+\lambda_{x y}^{\prime}\left(\partial_{x} h \partial_{y} h\right)-\nabla^{4} h \\
& -\partial_{x}\left[\partial_{x} h-\Omega\left(\partial_{x} h\right)^{3}\right]-\partial_{y}\left[\partial_{y} h-\Omega\left(\partial_{y} h\right)^{3}\right]
\end{aligned}
$$

with values for $\lambda_{x, y}$ obtained from MD simulations can describe the topographical patterns forming on a crystalline $\mathrm{Ge}(001)$ surface under low-energy ion irradiation in the reverse epitaxy regime well. With $\Omega_{3}=\Omega_{4}:=\Omega$ being constant as expected for a purely diffusive term, the observed morphological changes can be modeled by varying only $\lambda_{x, y}$ as predicted by MD simulations. The morphology of alternating pyramidal pits and mounds is reproduced, as are their specific deformations with increasing $\vartheta_{\text {ion }}$ for both of the azimuthal ion incidence directions. The dependence of the pattern morphology on $\vartheta_{\text {ion }}$ therefore appears to be predominantly an effect of the angle-dependent sputter yield. 
Comparing Figs. 2(f) and 2(1) and Figs. 3(f) and 3(1), some deviations from the experimental data are observed for $\vartheta_{\text {ion }}=$ $80^{\circ}$ : First, the simulated surface at $\varphi_{\text {ion }}=315^{\circ}$ exhibits a far more pronounced elongation of the pyramidal structures than the experimentally observed morphology. Second, the experimental data would have been modeled better by choosing $\lambda_{x}>0$ so as not to invert the topography. These deviations occur at very oblique ion incidence angles, where the sputter yield changes drastically with the ion incidence angle [32]. The coefficients $\lambda_{x, y}$ are determined from the first and second derivative of a Yamamura fit to the angle-dependent sputter yield. Therefore, their values for oblique angles of incidence are afflicted by significant uncertainty. Additional MD simulations covering the range of incidence angles from $70^{\circ}$ to $90^{\circ}$ in smaller increments would be required for reducing this uncertainty. Third, as can be inferred from the angle distributions in Figs. 2(k) and 2(1), the pyramids have an asymmetric profile along the ion beam direction for high polar angles of incidence. This is not reproduced in the simulations, though. The formation of similarly asymmetrically terraced morphologies on Ag single crystals was observed experimentally by Hauffe [47]. Pearson and Bradley [39] proposed to include higherorder nonlinear terms as corrections to the slope-dependent sputter yield, resulting in the formation of such terraced surfaces. Thus, including these terms in the continuum equation may resolve this difference between experiment and simulation seen here.

Some publications have dealt with applying a continuum equation approach to crystalline surfaces: $\mathrm{Ou}$ and co-workers have studied patterning of crystalline semiconductor surfaces by means of experiments and simulations based on a continuum equation which first included the term $\vec{j}_{\mathrm{ES}}$ for anisotropic diffusion $[14,21]$. Their work was explicitly limited to the case of normal incidence ion irradiation, and instead emphasized the implications of sample temperature, ion fluence, as well as lattice structure and orientation of the crystalline surface. The interplay of these factors with effects of off-normal irradiation may be worth further investigations. In very recent work, Chowdhury and Ghose discussed patterning of crystalline $\mathrm{Ge}(001)$ surfaces resulting from ion irradiation at off-normal incidence angles and an azimuthal orientation of $\varphi_{\text {ion }}=315^{\circ}$ [48]. While they investigated the pattern evolution with fluence and the effect of continuously rotating the sample, they did not approach their results from a theoretical viewpoint. Golubović and co-workers addressed pattern formation in both growth and erosion of crystalline surfaces from the theoretical perspective employing a continuum equation approach. By analytical and numerical means they studied coarsening dynamics, pattern morphology on surfaces of different lattice orientations, as well as motion of dislocations [49]. Regarding growth and erosion to be equivalent, the authors did not include the nonlinear term $\lambda_{x}\left(\partial_{x} h\right)^{2}+\lambda_{y}\left(\partial_{y} h\right)^{2}$ in their investigation at all. Since they did not state which incidence angle they were considering, it also remains unclear whether they generally presumed normal incidence or assumed that the angle of incidence has no influence on the resulting pattern. Renedo and co-workers [50] generalized the two-field model by Aste and Valbusa [51] to include anisotropic diffusion and studied the resulting surface morphologies under normal ion incidence. It should be of great interest to compare the results presented here with those of the approach by Renedo et al. for non-normal incidence, i.e., including anisotropic erosion.

\section{CONCLUSIONS}

We have prepared nanopatterned crystalline Ge(001) surfaces via low-energy ion irradiation in the reverse epitaxy regime at off-normal ion incidence angles and compared their topographies with corresponding simulated surface topographies. These were obtained from an anisotropic continuum equation with coefficients derived from molecular dynamics simulations. This anisotropic continuum equation including both anisotropic erosive and anisotropic diffusive effects proved highly suited for modeling the experimental results, using coefficients for angle-dependent erosion obtained from MD simulations. Only for near grazing ion incidence angles of $\vartheta_{\text {ion }} \geqslant 70^{\circ}$ did the simulated surface topographies deviate significantly from the experimentally observed ones. These deviations may be resolved by including higher-order nonlinear terms proportional to $\left(\partial_{x} h\right)^{3}$ and $\partial_{x} h\left(\partial_{y} h\right)^{2}$ as proposed by Pearson and Bradley to represent the formation of asymmetrically terraced morphologies. MD simulations could be used to estimate the coefficients of the respective terms. The main morphological changes resulting from irradiation at offnormal incidence can be reproduced by changing only the coefficients for angle-dependent sputter erosion, which shows the importance of this mechanism for ion-induced nanopattern formation on crystalline surfaces.

\section{ACKNOWLEDGMENTS}

We carried out all experimental parts of this research at the Ion Beam Center at the Helmholtz-Zentrum DresdenRossendorf e.V.-a member of the Helmholtz Association. K.N. and A.I. acknowledge the Academy of Finland project NANOIS Grant Number 1309731 and grants of computer capacity Grant Number hy3089 from the IT Centre for Science in Finland, CSC, and the Finnish Grid and Cloud Infrastructure (persistent identifier urn:nbn:fi:research-infras2016072533). R.M.B. acknowledges the support of the US National Science Foundation through Grant No. DMS1814941.
[1] F. Frost, R. Fechner, B. Ziberi, J. Völlner, D. Flamm, and A. Schindler, Large area smoothing of surfaces by ion bombardment: Fundamentals and applications, J. Phys.: Condens. Matter 21, 224026 (2009).

[2] R. M. Bradley and J. M. E. Harper, Theory of ripple topography induced by ion bombardment, J. Vac. Sci. Technol. A 6, 2390 (1988)
[3] G. Carter and V. Vishnyakov, Roughening and ripple instabilities on ion-bombarded Si, Phys. Rev. B 54, 17647 (1996).

[4] W. W. Mullins, Flattening of a nearly plane solid surface due to capillarity, J. Appl. Phys. 30, 77 (1959).

[5] J. G. Amar and F. Family, Step-Adatom Attraction as a New Mechanism for Instability in Epitaxial Growth, Phys. Rev. Lett. 77, 4584 (1996). 
[6] U. Valbusa, C. Boragno, and F. Buatier de Mongeot, Nanostructuring surfaces by ion sputtering, J. Phys.: Condens. Matter 14, 8153 (2002).

[7] W. L. Chan and E. Chason, Making waves: Kinetic processes controlling surface evolution during low energy ion sputtering, J. Appl. Phys. 101, 121301 (2007).

[8] F. Buatier de Mongeot and U. Valbusa, Applications of metal surfaces nanostructured by ion beam sputtering, J. Phys.: Condens. Matter 21, 224022 (2007).

[9] A. Keller and S. Facsko, Ion-induced nanoscale ripple patterns on Si surfaces: Theory and experiment, Materials 3, 4811 (2010).

[10] S. Rusponi, G. Costantini, C. Boragno, and U. Valbusa, Ripple Wave Vector Rotation on Anisotropic Crystal Sputtering, Phys. Rev. Lett. 81, 2735 (1998).

[11] W. L. Chan, N. Pavenayotin, and E. Chason, Kinetics of ioninduced ripple formation on $\mathrm{Cu}(001)$ surfaces, Phys. Rev. B 69 , 245413 (2004).

[12] H. Zhou, Y. Wang, L. Zhou, R. L. Headrick, A. S. Özcan, Y. Wang, G. Özaydin, K. F. Ludwig, Jr., and D. P. Siddins, Wavelength tunability of ion-bombardment-induced ripples on sapphire, Phys. Rev. B 75, 155416 (2007).

[13] M. Engler, S. Macko, F. Frost, and T. Michely, Evolution of ion beam induced patterns on $\mathrm{Si}(001)$, Phys. Rev. B 89, 245412 (2014)

[14] X. Ou, K.-H. Heinig, R. Hübner, J. Grenzer, X. Wang, M. Helm, J. Fassbender, and S. Facsko, Faceted nanostructure arrays with extreme regularity by self-assembly of vacancies, Nanoscale 7 , 18928 (2015).

[15] S. Facsko, T. Dekorsy, C. Koerdt, C. Trappe, H. Kurz, A. Vogt, and H. L. Hartnagel, Formation of ordered nanoscale semiconductor dots by ion sputtering, Science 285, 1551 (1999)

[16] F. Frost, A. Schindler, and F. Bigl, Roughness Evolution of Ion Sputtered Rotating InP Surfaces: Pattern Formation and Scaling Laws, Phys. Rev. Lett. 85, 4116 (2000).

[17] S. Le Roy, E. Søndergård, I. S. Nerbø, M. Kildemo, and M. Plapp, Diffuse-interface model for nanopatterning induced by self-sustained ion-etch masking, Phys. Rev. B 81, 161401(R) (2010)

[18] M. Ritter, M. Stindtmann, M. Farle, and K. Baberschke, Nanostructuring of the $\mathrm{Cu}(001)$ surface by ion bombardment, Surf. Sci. 348, 243 (1996).

[19] G. Costantini, S. Rusponi, R. Gianotti, C. Boragno, and U. Valbusa, Temperature evolution of nanostructures induced by $\mathrm{Ar}^{+}$sputtering on $\operatorname{Ag}(100)$, Surf. Sci. 416, 245 (1998).

[20] M. Fritzsche, A. Mücklich, and S. Facsko, Nanohole pattern formation on germanium induced by focused ion beam and broad beam $\mathrm{Ga}^{+}$irradiation, Appl. Phys. Lett. 100, 223108 (2012).

[21] X. Ou, A. Keller, M. Helm, J. Fassbender, and S. Facsko, Reverse Epitaxy of Ge: Ordered and Faceted Surface Patterns, Phys. Rev. Lett. 111, 016101 (2013).

[22] M. Engler and T. Michely, Amorphous to crystalline phase transition: Onset of pattern formation during ion erosion of Si(001), Phys. Rev. B 93, 085423 (2016).

[23] X. Ou, R. Kögler, X. Wei, A. Mücklich, X. Wang, W. Skorupa, and S. Facsko, Fabrication of horizontal silicon nanowire arrays on insulator by ion irradiation, AIP Adv. 1, 042174 (2011).
[24] B. Teshome, S. Facsko, and A. Keller, Topography-controlled alignment of DNA origami nanotubes on nanopatterned surfaces, Nanoscale 6, 1790 (2014).

[25] F. Bisio, C. Martella, L. Anghinolfi, M. C. Giordano, M. Carminale, M. Canepa, and F. Buatier de Mongeot, Plasmonics in self-organized media, in Encyclopedia of Nanotechnology, edited by B. Bushan (Springer Science+Business Media, Dodrecht, 2015).

[26] M. P. Harrison and R. M. Bradley, Fabrication of high quality multilayer blazed gratings using ion beam assisted deposition, J. Appl. Phys. 121, 225304 (2017).

[27] X. Ou, R. Hübner, X. Wang, J. Fassbender, and S. Facsko (unpublished)

[28] D. Nečas and P. Klapetek, Gwyddion: An open-source software for SPM data analysis, Cent. Eur. J. Phys. 10, 181 (2012).

[29] R. Cuerno and A.-L. Barabási, Dynamic Scaling of IonSputtered Surfaces, Phys. Rev. Lett. 74, 4746 (1995).

[30] M. Moseler, P. Gumbsch, C. Casiraghi, A. C. Ferrari, and J. Robertson, The ultrasmoothness of diamond-like carbon surfaces, Science 309, 1545 (2005).

[31] B. Davidovitch, M. J. Aziz, and M. P. Brenner, On the stabilization of ion sputtered surfaces, Phys. Rev. B 76, 205420 (2007).

[32] See Supplemental Material at http://link.aps.org/supplemental/ 10.1103/PhysRevB.102.165422 for details on the dependence of the sputter yield on the ion incidence angle as obtained from MD simulations, on the anisotropic variant of the continuum equation and its numerical integration, and for additional AFM data of Ge surfaces after irradiation at room temperature.

[33] R. M. Bradley, Dynamic scaling of ion-sputtered rotating surfaces, Phys. Rev. E 54, 6149 (1996).

[34] M. Kardar, G. Parisi, and Y.-C. Zhang, Dynamic Scaling of Growing Interfaces, Phys. Rev. Lett. 56, 889 (1986).

[35] J. Villain, Continuum models of crystal growth from atomic beams with and without desorption, J. Phys. I France 1, 19 (1991).

[36] Z. Zhang and M. G. Lagally, Atomistic processes in the early stages of thin-film growth, Science 276, 377 (1997).

[37] O. Pierre-Louis, M. R. D’Orsogna, and T. L. Einstein, Edge Diffusion during Growth: The Kink Ehrlich-Schwoebel Effect and Resulting Instabilities, Phys. Rev. Lett. 82, 3661 (1999).

[38] M. Siegert, Coarsening Dynamics of Crystalline Thin Films, Phys. Rev. Lett. 81, 5481 (1998).

[39] D. A. Pearson and R. M. Bradley, Theory of terraced topographies produced by oblique-incidence ion bombardment of solid surfaces, J. Phys.: Condens. Matter 27, 015010 (2015).

[40] M. Ghaly, K. Nordlund, and R. S. Averback, Molecular dynamics investigations of surface damage produced by $\mathrm{keV}$ self-bombardment of solids, Philos. Mag. A 79, 795 (1999).

[41] K. Nordlund, M. Ghaly, R. S. Averback, M. Caturla, T. Diaz de la Rubia, and J. Tarus, Defect production in collision cascades in elemental semiconductors and FCC metals, Phys. Rev. B 57, 7556 (1998).

[42] The open-source PARCAS code is available at gitlab, https:// gitlab.com/acclab/parcas/.

[43] K. Nordlund, Molecular dynamics simulation of ion ranges in the 1-100 keV energy range, Comput. Mater. Sci. 3, 448 (1995).

[44] Y. Yamamura, Y. Itikawa, and N. Itoh, IPPJ-AM-26: Angular Dependence of Sputtering Yields of Monoatomic Solids (Institute of Plasma Physics, Nagoya University, Nagoya, Japan, 1983). 
[45] A. Keller, S. Facsko, and R. Cuerno, Numerical integrator for continuum equations of surface growth and erosion, in Computational Nanotechnology: Modeling and Applications with MATLAB, edited by S. M. Musa (CRC Press, Boca Raton, FL, 2012), Chap. 5, pp. 189-215.

[46] M. Teichmann, J. Lorbeer, B. Ziberi, F. Frost, and B. Rauschenbach, Pattern formation on Ge by low energy ion beam erosion, New J. Phys. 15, 103029 (2013).

[47] W. Hauffe, Faceting mechanism in the sputtering process, Phys. Status Solidi A 35, K93 (1976).

[48] D. Chowdhury and D. Ghose, $\mathrm{Ar}^{+}$-sputtered Ge (001) surface nanostructuring at target temperature above the recrys- tallization threshold, J. Phys. D: Appl. Phys. 53, 335304 (2020).

[49] L. Golubović, A. Levandovsky, and D. Moldovan, Interface dynamics and far-from-equilibrium phase transitions in multilayer epitaxial growth and erosion on crystal surfaces: Continuum theory insights, E. Asian J. Appl. Math. 1, 297 (2011).

[50] J. Renedo, R. Cuerno, M. Castro, and J. Muñoz-García, Symmetry of surface nanopatterns induced by ion-beam sputtering: Role of anisotropic surface diffusion, Phys. Rev. B 93, 155424 (2016).

[51] T. Aste and U. Valbusa, Surface instabilities in granular matter and ion-sputtered surfaces, Physica A 332, 548 (2004). 